\title{
Meta-analysis: The Effect of Muscle Strength Training on Walking Ability of Patients with Parkinson's Disease
}

\author{
Meng Zhaoli ${ }^{1}$, Zhi Xiao ${ }^{1}$, Li Beibei ${ }^{1}$, Zheng Yanpei ${ }^{1}$, Li Lin ${ }^{2}$, Du Fei ${ }^{2}$, Li Tong ${ }^{2}$, Yan Zhi ${ }^{2,}$, \\ Liang Zhanghua ${ }^{3, *}$ \\ ${ }^{1}$ School of Kinesiology and Promotion, Dalian University of Technology, Dalian, China \\ ${ }^{2}$ Department of Neurology, Dalian Port Hospital, Dalian, China \\ ${ }^{3}$ Department of Neurology, the First Affiliated Hospital of Dalian Medical University, Dalian, China
}

Email address:

mengzl@dlut.edu.cn (Meng Zhaoli),Xiao.zhill@ foxmail.com (Zhi Xiao), 839654641@qq.com (Li Beibei), 1570664704@qq.com (Zheng Yanpei), lilin13478754655@126.com (Li Lin), dufei198813@126.com (Du Fei), hys907325@163.com (Li Tong), qiqiaojie@126.com (Yan Zhi), zhanhualiang@163.com (Liang Zhanghua)

${ }^{*}$ Corresponding author

\section{To cite this article:}

Meng Zhaoli, Zhi Xiao, Li Beibei, Zheng Yanpei, Li Lin, Du Fei, Li Tong, Yan Zhi, Liang Zhanghua. Meta-analysis: The Effect of Muscle Strength Training on Walking Ability of Patients with Parkinson's Disease. Rehabilitation Science. Vol. 6, No. 1, 2021, pp. 1-9. doi: $10.11648 /$ j.rs.20210601.11

Received: December 29, 2020; Accepted: January 14, 2021; Published: January 22, 2021

\begin{abstract}
Objective: This Meta-analysis was aimed to systematically studying the effects of muscle strength training on the walking ability like balance ability, mobility ability of Parkinson's disease patients and then provide useful theoretical reference to further researches on exercise treatments on Parkinson's disease by retrieving and collecting articles about muscle strength training. Methods This literature review was finally determined on searching PubMed, Elsevier, Web of science, China Journal Full-text Database (CNKI), WanFang Data and other Chinese and foreign databases and combined with manual search. The limit of the search time was from the date when the literature was recorded to 2019. A comprehensive collection of randomly controlled trials of muscle strength training on the walking ability of Parkinson's patients was done. Researchers used the Cochrance risk assessment tool to evaluate the methodological quality of the selected articles, and the ReMan 5.3.5 software to statistically process the obtained data. Results A total of 13 RCTs and 627 samples were included in this study. Meta-analysis of BBS balance scale show that MD=4.67 $\left(95 \% \mathrm{CI}, \mathrm{I}^{2}=97 \%, \mathrm{P}=0.52\right)$ between muscle strength training group and non-exercise intervention group; MD=-2.67 between muscle strength training group and exercise intervention control group $\left(95 \% \mathrm{CI}, \mathrm{I}^{2}=7 \%, \mathrm{P}<0.00001\right)$; TUGT Meta-analysis show that $\mathrm{MD}=-1.06\left(95 \% \mathrm{CI}, \mathrm{I}^{2}=75 \%, \mathrm{P}=0.10\right)$ between muscle strength training group and non-exercise intervention group; $\mathrm{MD}=0.09$ $\left(95 \% \mathrm{CI}, \mathrm{I}^{2}=0 \%, \mathrm{P}=0.31\right)$ between the muscle strength training group and the control group with exercise intervention; 10MWT Meta-analysis show $\left.\mathrm{MD}=-0.28(95 \% \mathrm{CI}), \mathrm{I}^{2}=98 \%, \mathrm{P}<0.29\right)$ between the muscle strength training group and the control group with exercise intervention; Stride length Meta-analysis show $\mathrm{MD}=-1.85\left(95 \% \mathrm{CI}, \mathrm{I}^{2}=68 \%, \mathrm{P}=0.63\right)$ between the muscle strength training group and the control group without exercise intervention; $\mathrm{MD}=-1.75\left(95 \% \mathrm{CI}, \mathrm{I}^{2}=32 \%, \mathrm{P}=0.56\right)$ between the muscle strength training group and the control group with exercise intervention; $\mathrm{MD}=-1.75\left(95 \% \mathrm{CI}, \mathrm{I}^{2}=32 \%, \mathrm{P}=0.56\right)$. Meta-analysis of stride speed show $\mathrm{MD}=-0.02\left(95 \% \mathrm{CI}, \mathrm{I}^{2}=0 \%, \mathrm{P}=0.46\right)$ between muscle strength training group and control group without exercise intervention; $\mathrm{MD}=-0.03\left(95 \% \mathrm{CI}, \mathrm{I}^{2}=35 \%, \mathrm{P}=0.52\right)$ between the muscle strength training group and control group with exercise intervention. Conclusion Muscle strength training can significantly improve the balance ability, mobility, and walking ability of Parkinson's disease patients, but it has no significant benefits on improving stride length and walking speed.
\end{abstract}

Keywords: Strength Training, Parkinson's Disease, Walking Ability

\section{Introduction}

Parkinson's disease (PD) is a kind of neurodegenerative disease, which is clinically manifested as akinesia (shortened step length, slower walking speed, often longer gait cycle time, increased step length variability and asymmetry), stiffness, tremors and unstable posture $[1,2]$. According to statistics, 
among people over the age of 50 , the incidence rate is $70.7 / 100,000$ [3], and the disability rate is high. It is reported that after 1 to 5 years of onset, the disability rate is $25 \%$, and it cloud reach $66 \%$ after 5-9 years [4] and gradually became younger trend. Due to the impairment of the central and peripheral nerve control ability, the patient's muscle strength is reduced, which has a great correlation with the patient's risk of falling and slower walking speed [5] leads to a decrease in the patient's balance ability and gait disturbance. The fall rate and the mortality rate increases in the course of the disease has seriously affected the patient's mobility and quality of life.

In recent years, exercise intervention has been widely studied as a non-medical method for clinical auxiliary treatment of Parkinson's disease patients, which can improve patients' balance ability, gait disturbance, increase muscle strength, and prevent falls $[6,7]$. Strength training is an intervention method to make effects on exercise through resisting resistance. Studies have proved that muscle strength training is an effective, safe and feasible exercise therapy that assists in the treatment of clinical features of patients with neurodegenerative diseases [8-10]. This research conducts a systematic review and meta-analysis of this type of related research to classify and analyze the clinical indicators of Parkinson's disease patients. According to the results, the study adopt subgroup analysis to summarize and evaluate the specific application of muscle training in clinical rehabilitation.

\section{Materials and Methods}

\subsection{Literature Retrieval}

PubMed, Elsevier, Web of science, China Journal Full-text Database (CNKI), WanFang Data and other Chinese and foreign databases with MeSH were retrieved. The keywords includes "parkinson disease", "parkinson's disease", "Strength trainging", "resistance trainging", etc. A total of 2490 relevant documents were obtained, and 1119 were selected after screening and deduplication according to the research direction. After reading the abstract, 127 articles were included. 95 articles variables did not meet the inclusion criteria, and the remaining 32 articles were read. The outcome indicators of the 19 articles could not be obtained in the form of mean \pm standard deviation $(\overline{\mathrm{X}} \pm S)$, and finally 13 articles were included in this meta-analysis.

\subsection{Inclusion Criteria}

The quality of the included research literature directly affects the reliability and validity of the meta-analysis results. In order to get qualified studies, there must be strict screening criteria: (1) The study of the influence of muscle strength training on the gait of patients with Parkinson's disease must be randomized clinical trials (RCT); (2) Patients who have been diagnosed with Parkinson's disease according to the British Brain Bank Parkinson's disease diagnostic criteria [10]; (3) The training method of the experimental group is strength training (including resistance training) while the control group received other exercise intervention therapy or blank control;
(4) The variables include BBS/Berg balance scale, stride length, walking speed, time up to go test (TUGT), and 10 -meter walk test (10 meters walking test, 10MWT); (5) Electronic full text is available.

\subsection{Data Information Extraction}

The content of data extraction includes: (1) Basic information of the literature, such as author, year of publication; (2) Trial design includes randomization, control, blinding, etc.; (3) General information of the research objects obtain age, number of research objects, intervention methods, total intervention time, outcome indicators, etc. If the included RCT in the literature involves multiple grouping studies, extract the experimental group and the control group that are consistent with the purpose of this research. The data is showed in the form of mean \pm standard deviation $(\bar{X} \pm S)$.

\subsection{Statistical Analysis}

RevMan 5.3.3 statistical software was used for bias assessment, heterogeneity test, data merging, and drawing bias and forest maps. The data processed in this study was continuous data, and the $95 \%$ confidence interval of MD (Mean Differences) random effects model. (1) Heterogeneity test: Use $\mathrm{I}^{2}$ to test heterogeneity, and $\mathrm{I}^{2}$ test to judge the degree of heterogeneity. When $\mathrm{P}>0.1$ and $\mathrm{I}^{2} \leq 50 \%$, the heterogeneity between the studies is considered to be small; when $\mathrm{P} \leq 0.1, \mathrm{I}^{2}>50 \%$, the heterogeneity between the studies is considered to be greater. Low heterogeneity chooses fixed effects model (FEM) analysis, and high heterogeneity chooses random effect model (REM).

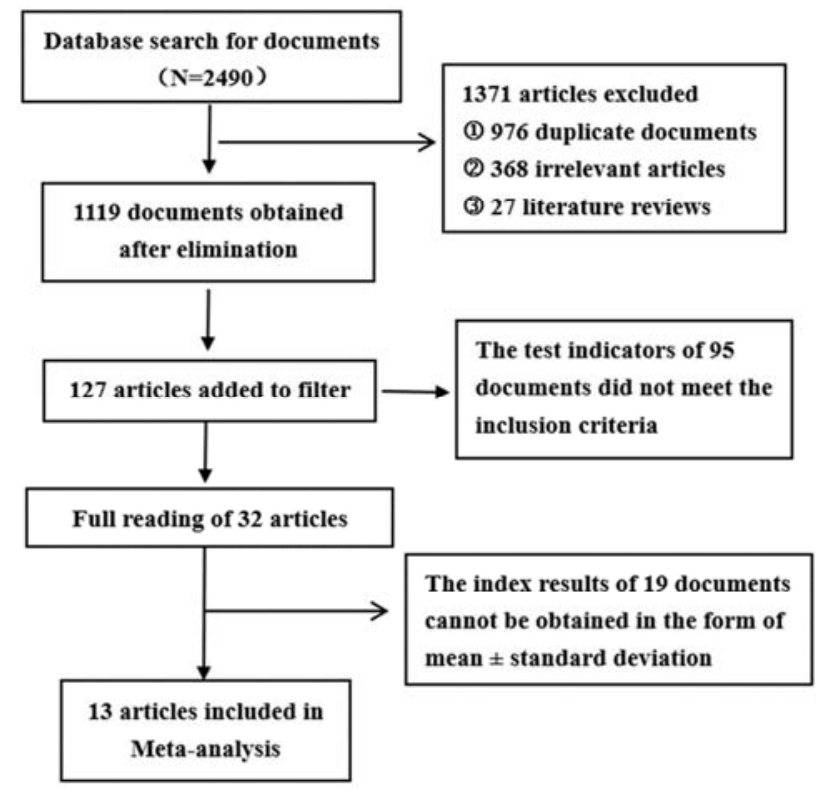

Figure 1. Screening Diagram of included literatures.

\section{Results and Analysis}

\subsection{Discussion on Characteristics and Quality of Included Literature}

A total of $13[6,11-22]$ articles on the influence of muscle 
strength training on walking ability of Parkinson's disease were included in this study, and the research period was from 2013 to 2019. There were 5 Chinese literatures and 9 foreign literatures, all of them were randomized controlled trials. There were 6 studies for the experimental group to receive strength training, the control group only received drug therapy as a blank control. In 7 studies, the experimental group received strength training, the control group received another exercise interventions include balance training, aerobic training and treadmill training. A total of 627 patients aged from 40 to 77 years old were recruited in this study. The duration of intervention period was more than 8 weeks. (Table 1).

From the overall point of view of Table 1, there is bigger difference of age among subjects included in the literature (from 40 to 70 years old), which is mainly reflected in the research of Tang Jie et al. From the perspective of intervention duration, there were also some differences. Miriam et al. carried out a two-year follow-up survey on the strength and improved fitness program of patients with Parkinson's disease, which was significantly longer than other researches periods.
In addition, the loss rate of Parkinson's disease patients from recruitment to the end of the experiment was relatively high, which may lead to high risk of data integrity. These results may be the heterogeneous sources of data bias.

\subsection{Assessment of Literature Bias}

In this study, Cochrane Handbook (5.1.0 version) was used to comprehensively evaluate the literature quality [23]. Figure 2 shows the bias evaluation chart of the included literature, and Figure 3 shows the risk summary chart of the included literature. Bias evaluation mainly included the following aspects: (1) random allocation method. (2) allocation concealment. (3) implementation of blind method. (4) integrity of results data. (5) selective reporting of research results. (6) other biases. The evaluation of literature quality is based on the handbook standard: "unclear" or "low risk" or "high risk". It can be seen from Figure 2 and Figure 3 that there are some bias in the literature included in the analysis, but the overall quality of the literature was above medium.

Table 1. Basic characteristics of included literature.

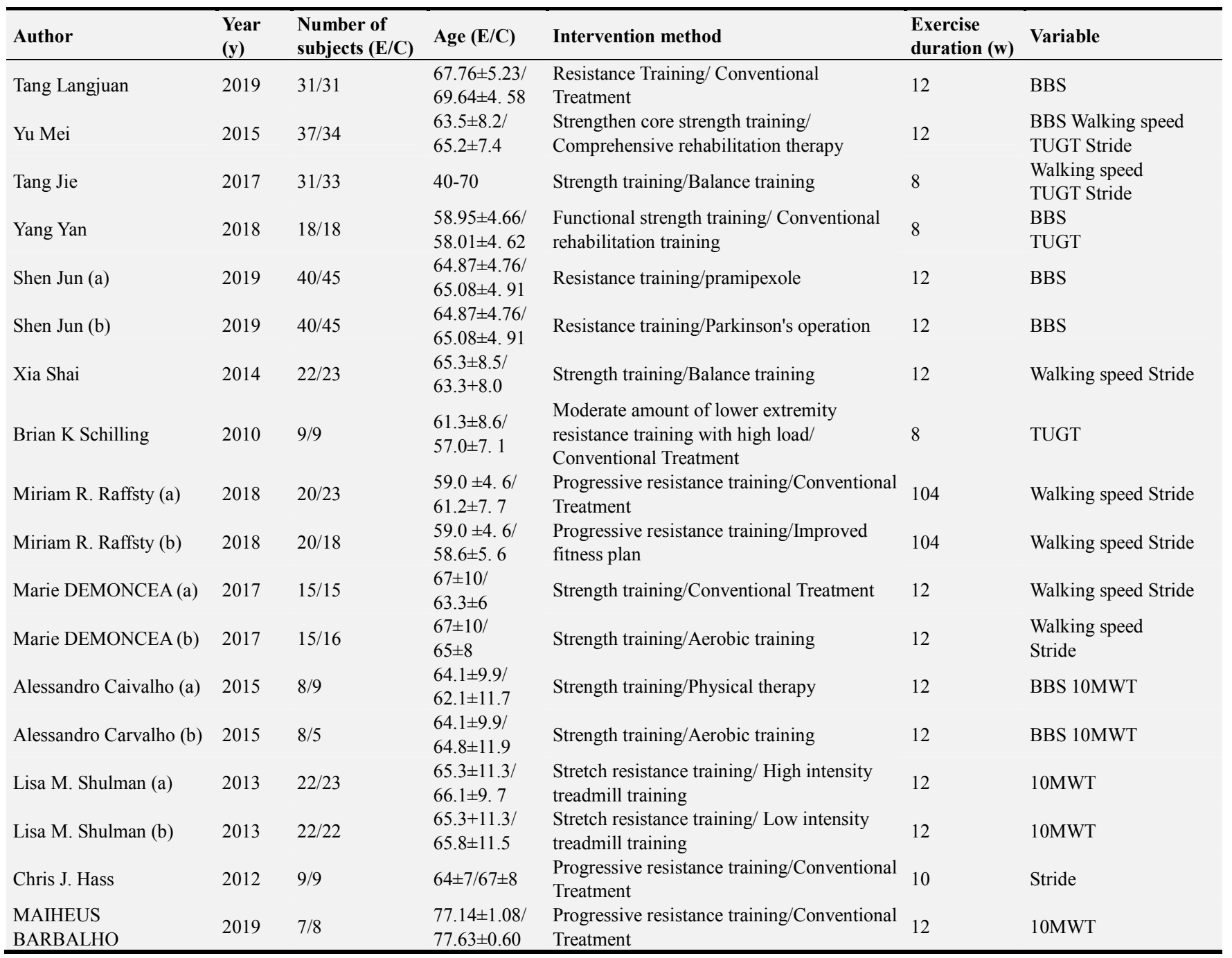

$\mathrm{E} / \mathrm{R}$ : $\mathrm{E}=$ experiment, $\mathrm{C}=$ control 


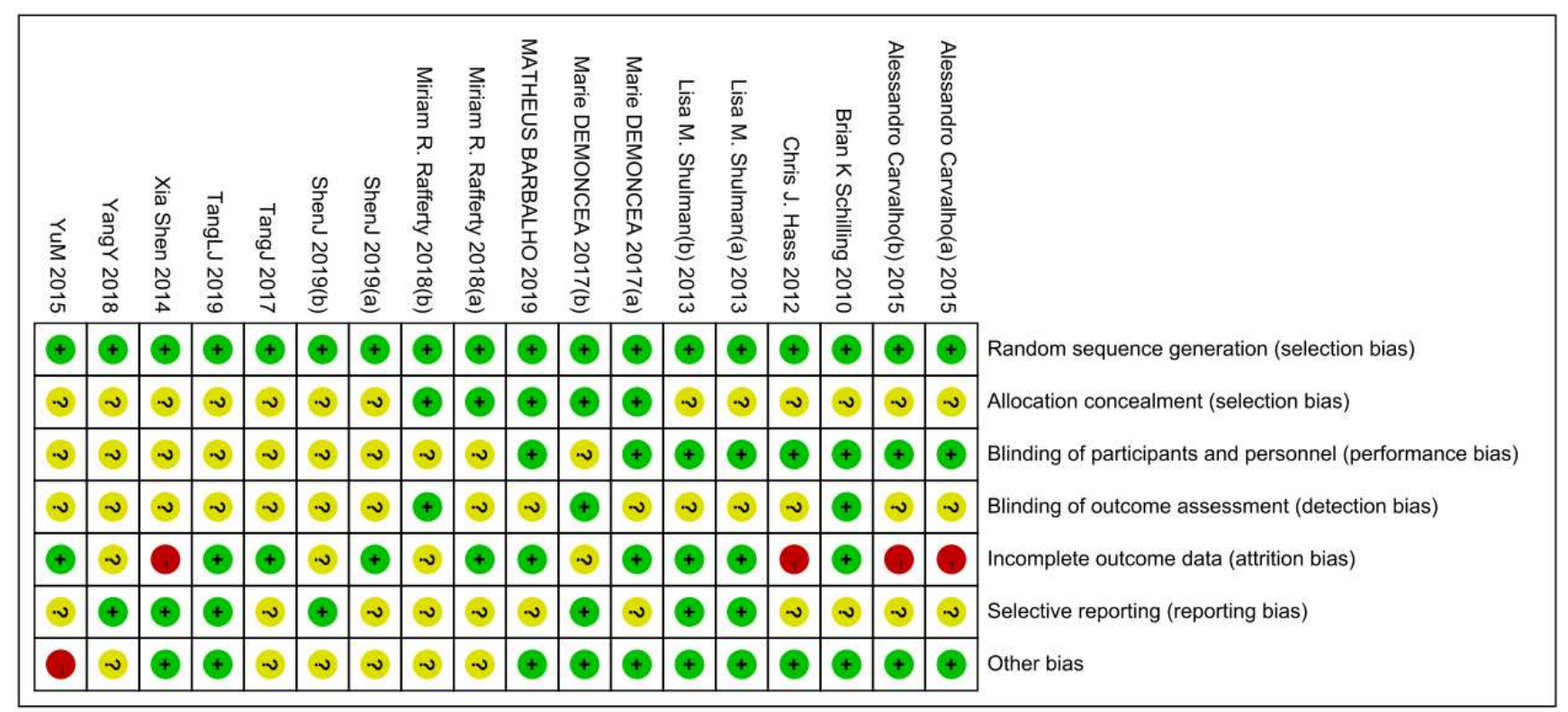

Figure 2. Schematic diagram of bias risk evaluation.

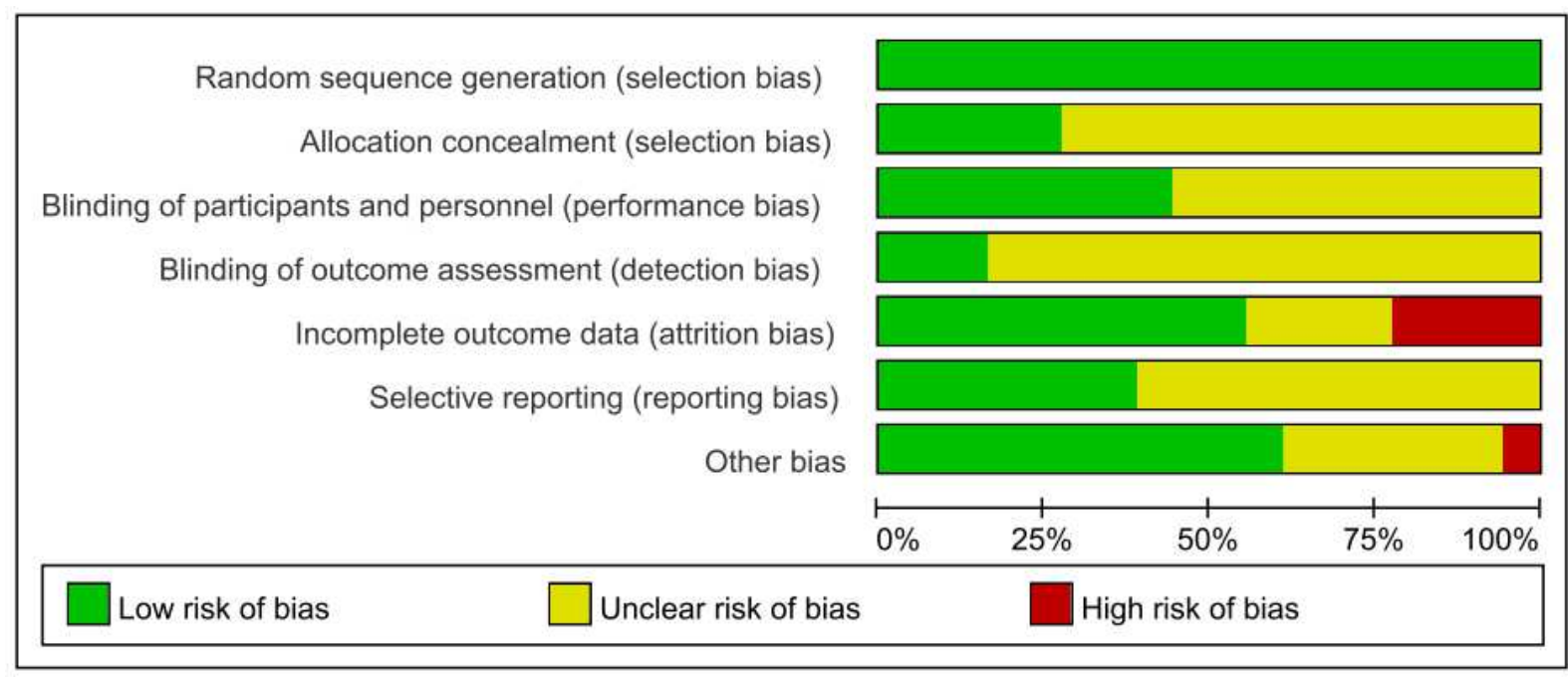

Figure 3. The ratio of risk of bias.

\subsection{Subgroup Analysis}

According to the difference of research design, the literature is divided into two categories. (1) Strength training group (experimental group) versus non exercise intervention group (control group); (2) strength training group (experimental group) versus exercise intervention group (control group). The influence of strength training on balance ability and motor ability of patients with Parkinson's disease was discussed by subgroup analysis.

\subsection{Main Results of Meta-analysis}

\subsubsection{Meta-analysis of BBS Balance Scale}

As shown in the meta-analysis of BBS balance scale in figure 4 and figure 5, the combined data analysis of experimental group and non exercise intervention group showed heterogeneity $\left(\mathrm{I}^{2}=99.64, \mathrm{P}<0.00001, \mathrm{I}^{2}=97 \%\right), 95 \%$ total effect confidence interval $1.67[-3.41,6.75], \mathrm{P}=0.52$. And the combined data analysis of the experimental group and the control group with exercise intervention showed heterogeneity $\left(\mathrm{I}^{2}=2.16, \mathrm{P}=0.34, \mathrm{I}^{2}=7 \%\right), 95 \%$ total effect confidence interval $-2.67[-3.68,-1.65], \mathrm{P}<0.00001)$.

\subsubsection{TUGT Meta-analysis}

Figure 6, the combined data analysis of the experimental group and the non exercise intervention group showed heterogeneity $\left(\mathrm{I}^{2}=12.10, \mathrm{P}=0.007, \mathrm{I}^{2}=75 \%\right), 95 \%$ total effect confidence interval $-1.06[-2.32,0.20], \mathrm{P}=0.10$. And the combined data analysis of the experimental group and the control group with exercise intervention showed heterogeneity $\left(\mathrm{I}^{2}=0.19, \mathrm{P}=0.67, \mathrm{I}^{2}=0 \%\right), 95 \%$ total effect confidence interval $0.09[-0.08,0.27], \mathrm{P}=0.31$. 


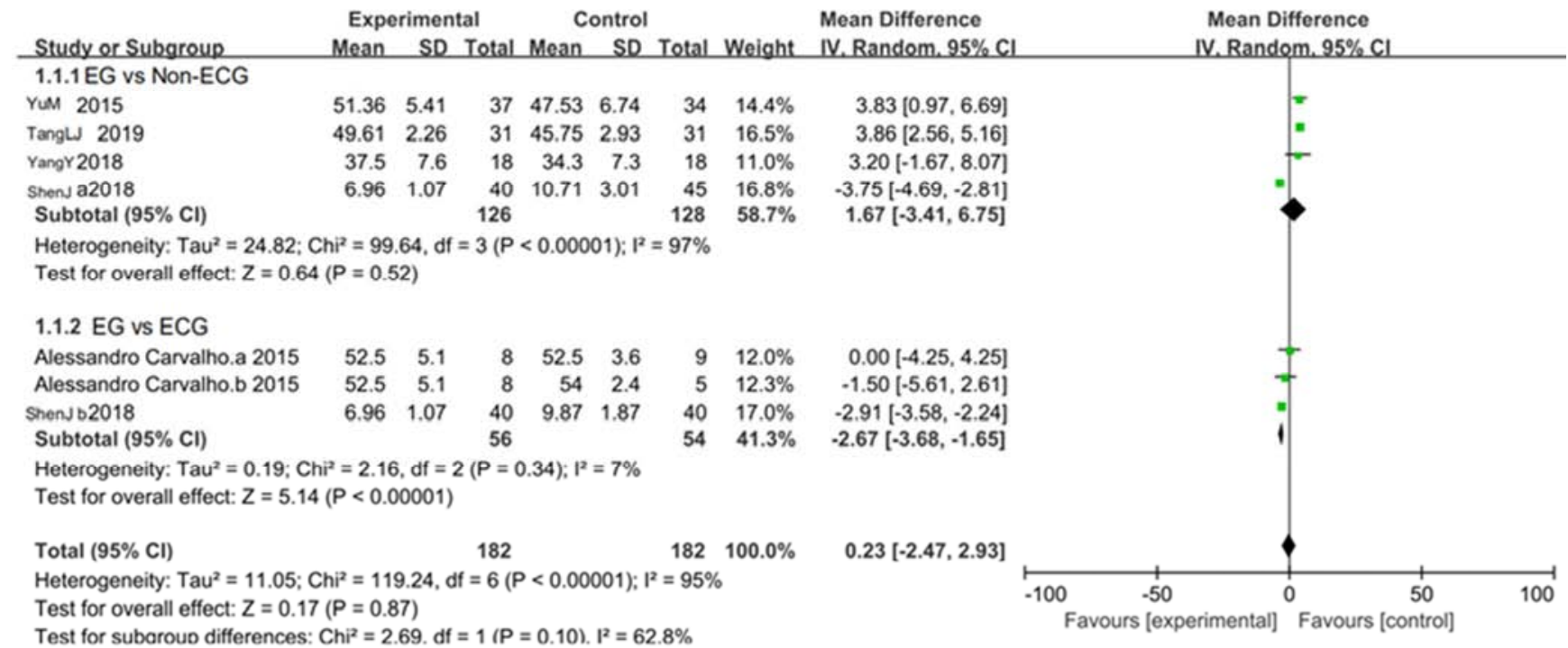

Figure 4. Meta-analysis forest diagram of BBS balance scale (1).

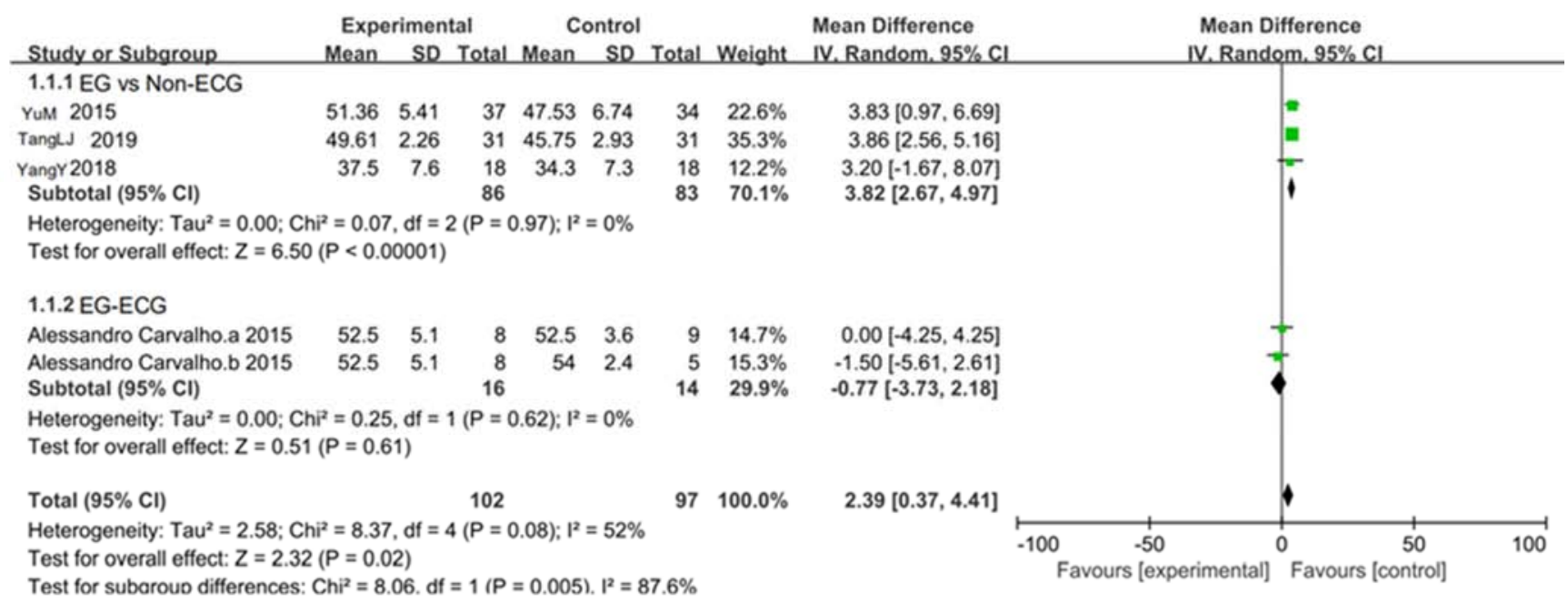

Figure 5. Meta-analysis forest diagram of BBS balance sacle (2).

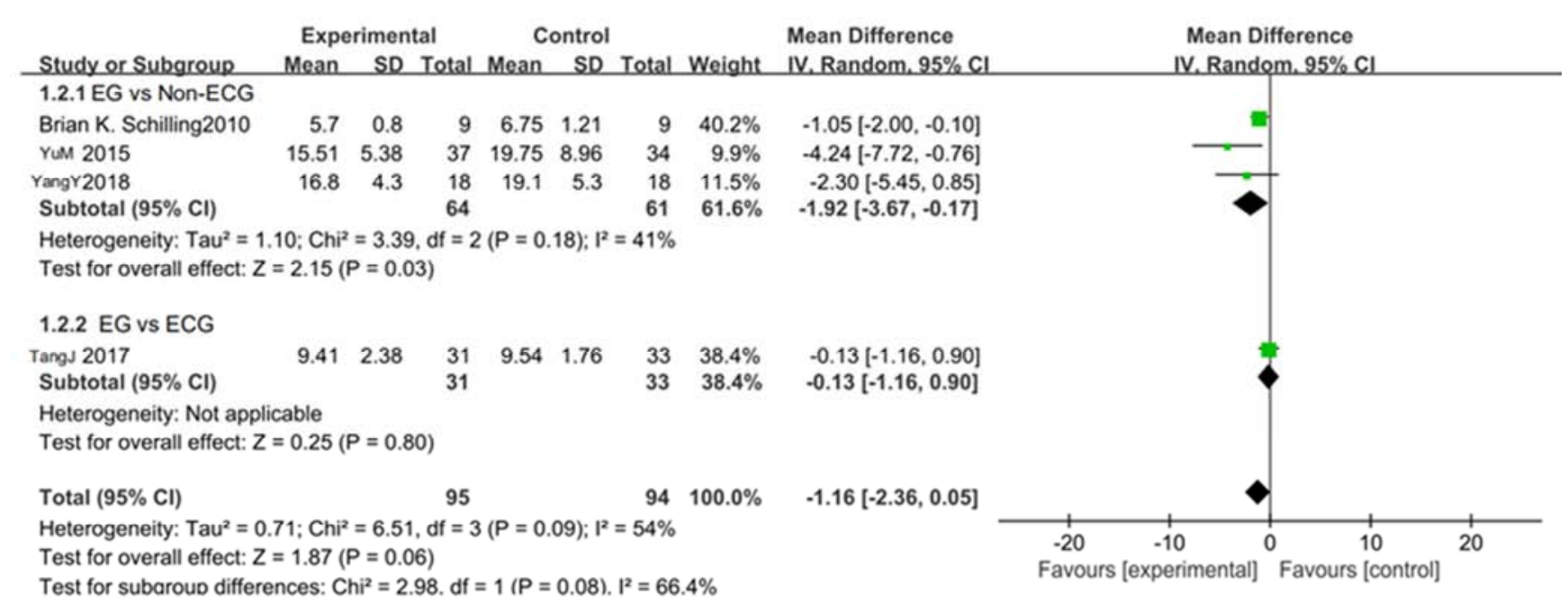

Figure 6. TUGT Meta-analysis forest map. 


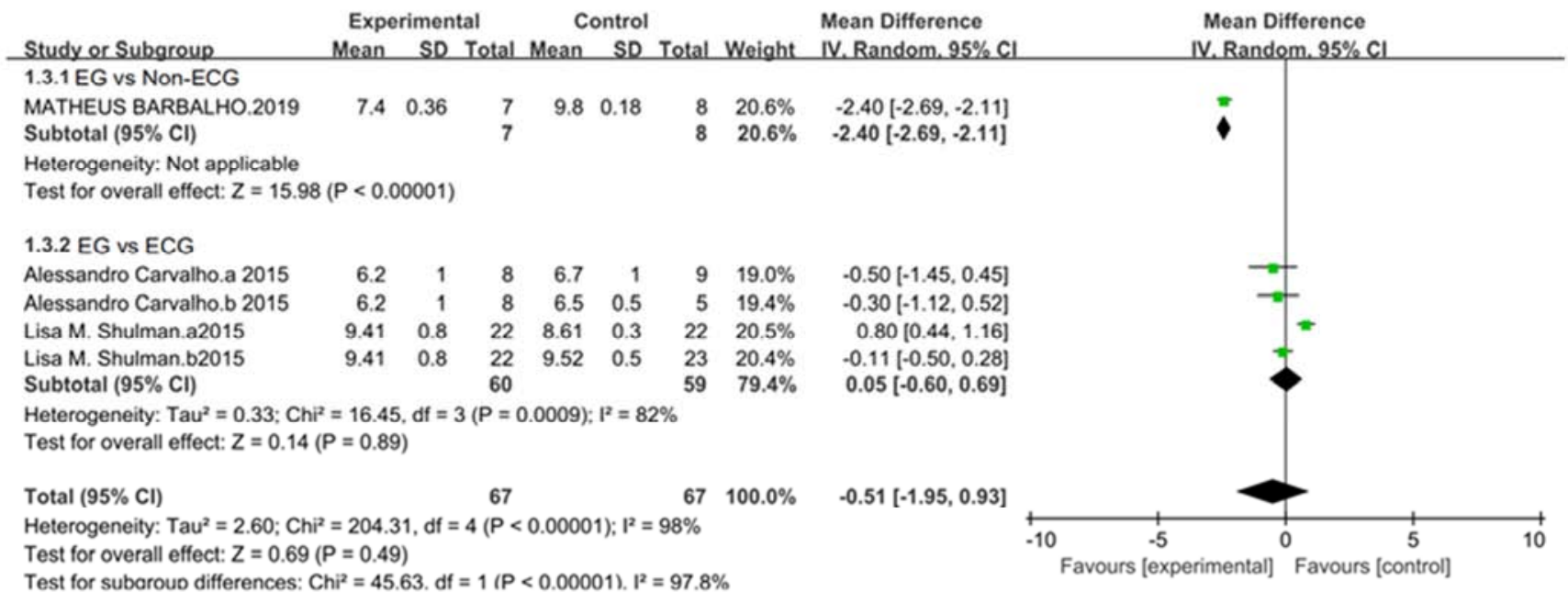

Figure 7. 10MWT Meta-analysis forest map.

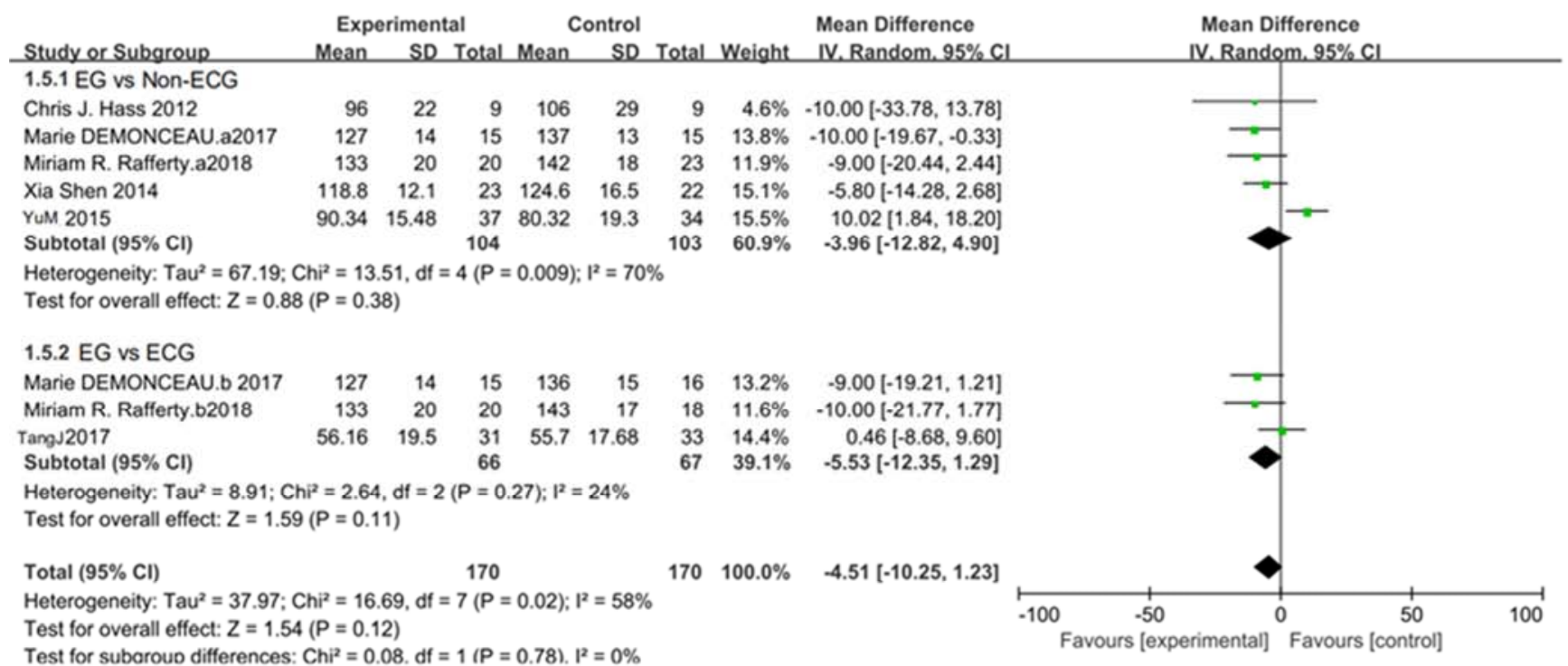

Figure 8. Stride Meta-analysis Forest Map.

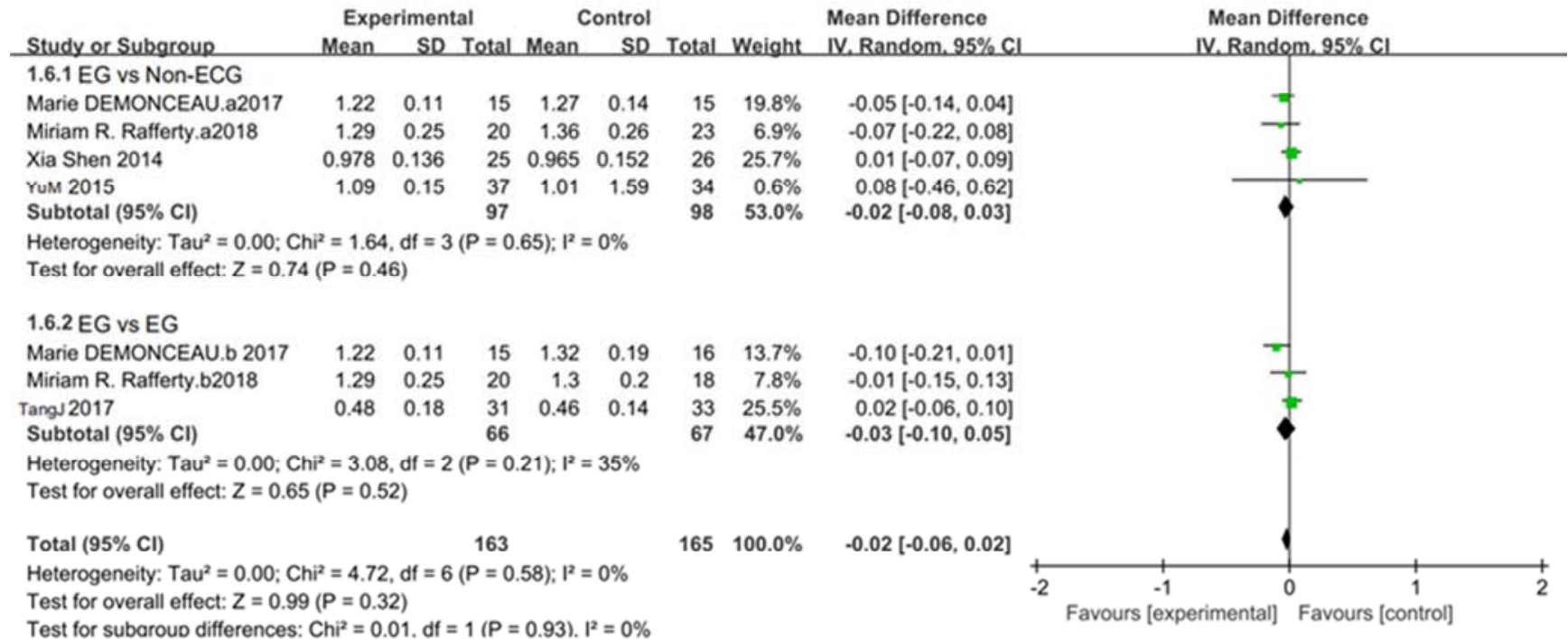

Figure 9. Meta-analysis forest diagram of pace. 


\subsubsection{MWT Meta-analysis}

As shown in Figure 7, the combined data analysis of the experimental group and the control group with exercise intervention showed heterogeneity $\left(\mathrm{I}^{2}=138.96, \mathrm{P}<0.00001\right.$, $\left.\mathrm{I}^{2}=98 \%\right), 95 \%$ total effect confidence interval $-0.82[-2.36$, $-0.71], \mathrm{P}<0.00001$.

\subsubsection{Meta-analysis of Stride Length}

In Figure 8, the combined data analysis of the experimental group and the control group with non exercise intervention showed heterogeneity $\left(\mathrm{I}^{2}=12.61, \mathrm{P}=0.01, \mathrm{I}^{2}=68 \%\right), 95 \%$ total effect confidence interval $-1.85[-9.27,5.57], \mathrm{P}=0.63$. And the combined data analysis of the experimental group and the control group with exercise intervention showed heterogeneity $\left(\mathrm{I}^{2}=2.93, \mathrm{P}=0.23, \mathrm{I}^{2}=32 \%\right), 95 \%$ total effect of confidence interval $-1.75[-7.59,4.10], \mathrm{P}=0.56$.

\subsubsection{Meta-analysis of Walking Speed}

As shown in Figure 9, the combined data analysis of the experimental group and the control group with non exercise intervention showed heterogeneity $\left(\mathrm{I}^{2}=1.64, \mathrm{P}=0.65, \mathrm{I}^{2}=0 \%\right)$, $95 \%$ total effect confidence interval $-0.02[-0.08,0.03]$, $\mathrm{P}=0.46$. And the combined data analysis of the experimental group and the control group with exercise intervention showed heterogeneity $\left(\mathrm{I}^{2}=3.08, \mathrm{P}=0.21, \mathrm{I}^{2}=35 \%\right), 95 \%$ total effect confidence interval $-0.03[-0.10,0.05], \mathrm{P}=0.52$.

\section{Discussion}

Weakened muscle strength leads to slower walking speed, weaker postural control ability and mobility in Parkinson's disease patients [24-25], which is also one of the independent factors leading to falls among the elderly people [26]. Amyotrophy caused by dopamine levels decline in Parkinson's disease leads to less stimulation through the basal ganglion-muscle or nerve-muscle pathway. In addition, most patients with Parkinson's disease are living a sedentary lifestyle and that is also a major cause of Amyotrophy. Muscle strength training can increase the nerve driving force by generating stimulation, as a result of recruiting more motor units (increase of motor muscle fibers) in order to increase muscle strength so that preventing fall [27] when patients are prone to. The results of this study showed that, compared with the control group without exercise intervention, muscle strength training had no effect on the balance ability of PD patients. After the inclusion [19] was eliminated according to the method of exclusion literature one by one, the result was positive $(\mathrm{P}<0.00001$, Figure 5). Compared with exercise intervention in the control group, there was no significant improvement through strength training than aerobic training and Parkinson's exercise training on the BBS score $(\mathrm{P}=0.61$, figure 4), the reasons for this result may be participants selected in the study of [19] had lower BBS score compared to other researchers at baseline. So a conclusion can illustrate that the study may be the heterogeneous sources of BBS subgroup analysis. TUGT and 10MWT respectively reflect the mobility of patients (the ability to perform other activities from sitting position to standing position and then to walking) and mobility. These two tests are widely used as the effectiveness and sensitivity indexes to evaluate patients' ability of activities of daily life [28-31]. The results of TUGT and 10MWT Meta-analysis showed that muscle strength training improved the mobility and walking ability of PD patients and meanwhile reduced the risk of falls of them to a certain extent, which were consistent with the results of Leland E. Dibble et al [32]. As for stride length, walking speed subgroup meta-analysis, muscle strength exercise group compared with control group without intervention and the control group with intervention, these two numbers of values did not change significantly before and after the intervention. According to N. E. Allen's mentioned in the study, the proper strength training can improve the muscle strength of patients with PD, there are also researchers believe that strength training is a kind of muscle power training that is the way to train the ability of fast muscle strength output. Lower muscle power output is also the reason why the elderly walk slow when they are aged.

Motor unit is mainly composed of two different kinds of motor units, type I and type II. The contraction speed of Type I motor unit is slower but not easy to fatigue, while type II has reverse characters compared type I, Type I units are raised first when muscles start to work and then type II units would be recruited if movements need more muscles to stimulate in order to quickly produce stronger power to complete the muscle activity. Strength training is mainly to increase muscle strength, and muscle output power training is to improve the ability to produce more strength quickly. However, the effectiveness of muscle strength training increase the ability of PD patients' movement speed has not been confirmed [26]. Therefore the influence of muscle strength training on walking speed and stride length needs to be further clarified.

\section{Conclusion}

In summary, muscle strength training significantly improved the balance ability and mobility of PD patients, but it is not seen that obvious improvements on stride length and walking speed. The duration of practice was shorter in some articles and the true effect on rehabilitation in patients may not have been fully manifested.

In this Meta-analysis, there are also some differences in the number of subjects, intervention methods and intervention duration among the included articles. Thus more high-quality literatures should be included for classification study, so as to further verify the impact of muscle strength training on walking ability of PD patients.

\section{Acknowledgements}

This work was supported by National key R \& D projects (2019YFF0301805) and the Natural Science Foundation Guidance Project of Liaoning Province, China (2019-ZD-1000, 2019-ZD-1002). 


\section{References}

[1] Peterson D S, Horak F B. Neural control of walking in people with Parkinsonism [J]. Physiology. 2016, 31 (2): 95.

[2] Wang Xinde. Diagnostic criteria and differential diagnosis of Parkinson's disease and Parkinson's syndrome [J]. Clinical Focus, 1986 (05): 21.

[3] Miao Hongshi. Theory and Practice of Rehabilitation Medicine [M]. Shanghai Scientific \& Technical Publishers, 2013.

[4] Moreland J D, Richardson J A, Goldsmith C H, et al. Muscle weakness and falls in older adults: a systematic review and meta-analysis [J]. Journal of the American Geriatrics Society. 2010, 52 (7): 1121-1129.

[5] Fuzhong L, Peter H, Kathleen F, et al. Tai chi and postural stability in patients with Parkinson's disease [J]. N Engl J Med. 2012, 366 (6): 511-519.

[6] Hass C J, Buckley T A, Pitsikoulis C, et al. Progressive resistance training improves gait initiation in individuals with Parkinson's disease [J]. Gait \& Posture. 2012, 35 (4): 669-673.

[7] Kj Lhede T, Vissing K, Dalgas U. Multiple sclerosis and progressive resistance training: a systematic review $[\mathrm{J}]$. Multiple Sclerosis. 2012, 18 (9): 1215-1228.

[8] Falvo M J, Schilling B K, Earhart G M. Parkinson's disease and resistive exercise: rationale, review, and recommendations [J]. Movement Disorders Official Journal of the Movement Disorder Society. 2010, 23 (1): 1-11.

[9] Lima, Lidiane O, Rodriguesdepaula, et al. Recruitment rate, feasibility and safety of power training in individuals with Parkinson's disease: a proof-of-concept study [J]. Revista Brasileira De Fisioterapia. 2013, 17 (1): 49-56.

[10] Hughes A J, Daniel S E, Kilford L, et al. Accuracy of clinical diagnosis of idiopathic Parkinson's disease: a clinico-pathological study of 100 cases [J]. Journal of Neurology Neurosurgery \& Psychiatry. 1992.

[11] Shen X, Mak M K Y. Balance and Gait Training With Augmented Feedback Improves Balance Confidence in People With Parkinson's Disease [J]. Neurorehabilitation and Neural Repair. 2014, 28 (6): 524-535.

[12] Alessandro Carvalho A, Barbirato D, Santos T, et al. Comparison of strength training, aerobic training, and additional physical therapy as supplementary treatments for Parkinson\&rsquo; $\mathrm{s}$ disease: pilot study $[\mathrm{J}]$. Clinical Interventions in Aging. 2015: 183.

[13] Rafferty M R, Prodoehl J, Robichaud J A, et al. Effects of 2 Years of Exercise on Gait Impairment in People with Parkinson Disease [J]. Journal of Neurologic Physical Therapy. 2017, 41 (1): 21-30.

[14] Barbalho M, Monteiro E P, Costa R R, et al. Effects of Low-Volume Resistance Training on Muscle Strength and Functionality of People with Parkinson's Disease [J]. Int J Exerc Sci. 2019, 12 (3): 567-580.

[15] Schilling B K, Pfeiffer R F, Ledoux M S, et al. Effects of Moderate-Volume, High-Load Lower-Body Resistance Training on Strength and Function in Persons with Parkinson's
Disease: A Pilot Study [J]. Parkinson's Disease. 2010, 2010: $1-6$.

[16] Demonceau M, Maquet D, Jidovtseff B, et al. Effects of twelve weeks of aerobic or strength training in addition to standard care in Parkinson's disease: a controlled study [J]. European journal of physical and rehabilitation medicine. 2017, 53 (2): 184.

[17] Shulman L M, Katzel L I, Ivey F M, et al. Randomized Clinical Trial of 3 Types of Physical Exercise for Patients with Parkinson Disease [J]. JAMA Neurology. 2013, 70 (2): 183.

[18] Yang Yan, Wang Sheng, Gu Zhaohua, Efficacy of post-pulling with functional muscle training on the balance function of patients with Parkinson's disease [J]. Chinese Journal of Rehabilitation, 2018, 33 (06): 502-504.

[19] Shen Jun, Shi Yongwei, Ju Qiangguo, et al. Progressive Resistance Training Combined with Pramipexole Therapy for Improving Muscular Tension in Patients with Parkinson's Disease [J]. Neural Injury and Functional Reconstruction 2019, 14 (03): 124-127.

[20] Tang Langjuan, Mei Gui, Wang Zhenzhen, et al. The effect of resistance training on improving lower limb muscle strength and balance function of patients with Parkinson's disease [J]. Chinese Journal of Gerontology, 2019, 39 (01): 127-130.

[21] Tang Jie, Zeng Yan, Lin Biying. Effects of strength and balance training on motor and posture control function in patients with Parkinson's disease [J]. Neural Injury and Functional Reconstruction, 2017, 12 (03): 266-268.

[22] Yu Mei, Li Liantao, Dong Tongbao, et al. The effect of strengthening core strength training on the rehabilitation of Parkinson's disease [J]. Guangdong Medical Journal, 2015, 36 (01): 77-79.

[23] Higgins J P S G. Cochrane Handbook for Systematic Reviews of Interventions [J]. 2008.

[24] Kakinuma S, Nogaki H, Pramanik B, et al. Muscle weakness in Parkinson's disease: isokinetic study of the lower limbs [J]. European Neurology. 1998, 39 (4): 218-222.

[25] Allen N E, Sherrington C. Reduced muscle power is associated with slower walking velocity and falls in people with Parkinson's disease [J]. Parkinsonism Relat Disord. 2010, 16 (4): 261-264.

[26] Latt M D, Lord S R, Morris J G L, et al. Clinical and physiological assessments for elucidating falls risk in Parkinson's disease [J]. Movement Disorders. 2010, 24 (9): 1280-1289.

[27] Intzandt B. Can a Power Training Program Reduce Fall Risk Factors in Parkinson's Disease? [D]. Wilfrid Laurier University, 2016.

[28] Jin Tingjian, Ye Xiangming, Linjian, et al. The effect of intensive weight-bearing exercise especially for affected lower limb on balance and walking function of stroke patients [J]. China Journal of rehabilitation medicine. 2009, 24 (11): 995-998.

[29] Alexandre, Tiago S, Meira, et al. Accuracy of Timed Up and Go Test for screening risk of falls among community-dwelling elderly [J]. Revista Brasileira De Fisioterapia. 2012, 16 (5): 381. 
[30] Campbell C M, Rowse J L, Ciol M A, et al. The Effect of Cognitive Demand on Timed Up and Go Performance in Older Adults with and Without Parkinson Disease [J]. Journal of Neurologic Physical Therapy. 2003, 27 (1): 2-7.

[31] Lang J T, Kassan T O, Devaney L L, et al. Test-Retest Reliability and Minimal Detectable Change for the 10-Meter Walk Test in Older Adults with Parkinson's disease [J]. Journal of Geriatric Physical Therapy. 2016, 39 (4).
[32] Dibble L E, Hale T F, Marcus R L, et al. High intensity eccentric resistance training decreases bradykinesia and improves quality of life in persons with Parkinson's disease: A preliminary study [J]. Parkinsonism Relat Disord, 2009, 15 (10): 752-757. 